\title{
Hyperbolic Valued Dislocated Metric Spaces
}

\author{
Nilay Değirmen ${ }^{1 *}$ \\ $1^{*}$ Ondokuz Mayis University, Faculty of Science and Arts, Department of Mathematics, Samsun, Turkey, (ORCID: 0000-0001-8192-8473), nilay.sager@omu.edu.tr
}

(1st International Conference on Applied Engineering and Natural Sciences ICAENS 2021, November 1-3, 2021)

(DOI: 10.31590/ejosat.1013323)

ATIF/REFERENCE: Değirmen, N. (2021). Hyperbolic Valued Dislocated Metric Spaces. European Journal of Science and Technology, (28), 1152-1158.

\begin{abstract}
In this work, we introduce the concept of a hyperbolic valued dislocated metric space, and we show that a fixed point of self-mappings in such spaces is exist and unique by using Banach, Kannan and Chatterjea type contractions. Furthermore, we discuss an illustrative example to substantiate the applicability and usefulness of our main result.
\end{abstract}

Keywords: Hyperbolic number, dislocated metric space, fixed point, contraction mapping.

\section{Hiperbolik Değerli Dislocated Metrik Uzaylar}

Öz

Bu çalışmada, hiperbolik değerli dislocated metrik uzay kavramını sunduk ve Banach, Kannan ve Chatterjea tip daralmaları kullanarak böyle uzayların kendileri üzerinde tanımlı dönüşümler için bir sabit noktanın var ve tek olduğunu gösterdik. Ayrıca ana sonucumuzun uygulanabilirliğini ve kullanışlılığını ispatlamak için açıklayıcı bir örnek verdik.

Anahtar Kelimeler: Hiperbolik sayı, dislocated metrik uzay, sabit nokta, daralma dönüşümü.

\footnotetext{
${ }^{*}$ Corresponding Author: nilay.sager@omu.edu.tr
} 


\section{Introduction}

Fixed point theory has huge applications in mathematics, computer science, engineering, image processing, economics, and statistics. The main goal of researchers is to extend the Banach contraction principle (Banach, 1922) with new contractive conditions in different metric spaces.

In 2000, as a generalization of metric spaces, Hitzler and Seda (2000) defined the concept of a dislocated metric space in which self distance of a point need not be equal to zero. They also stated the celebrated Banach contraction principle in such spaces. The study of fixed points of self-mappings in dislocated metric space is an active part of research in topology, electronic engineering and logic programming with wide range of applications in various directions. For several significant studies, see (Pasicki, 2015; Rahman and Sarwar, 2015; Wadkar et al., 2017; Gaba and Garg, 2019).

Recently, Kumar and Saini (2016) presented the concept of a hyperbolic valued metric space. In 2021, Sager and Sağır (2021) introduced some fixed point theorems for hyperbolic valued metric spaces by defining hyperbolic contraction mapping. Also, Sager and Sağır (2021) proved the existence of unique common fixed point for contraction mappings and a coupled coincidence and unique common coupled fixed point for two mappings on hyperbolic valued metric spaces.

The main aim of this study is to introduce the notion of a hyperbolic valued dislocated metric space and to give some fixed point theorems for our newly obtained contractions that are inspired from the results of Banach (1922), Kannan (1968) and Chatterjea (1972) on such spaces.

\section{Material and Method}

In this section, we give some necessary definitions and known results from recent literature.

Let $i$ and $j$ be independent imaginary units such that $i^{2}=j^{2}=-1, \quad i j=j i$. The set of bicomplex numbers $\mathrm{BC}$ is defined by

$$
\mathrm{BC}=\left\{z=z_{1}+j z_{2}: z_{1}, z_{2} \in \square\right\} .
$$

The set $\mathrm{BC}$ is a Banach space with respect to the addition, scalar multiplication and Euclidean norm defined as

$$
\begin{aligned}
z+w & =\left(z_{1}+j z_{2}\right)+\left(w_{1}+j w_{2}\right)=\left(z_{1}+w_{1}\right)+j\left(z_{2}+w_{2}\right), \\
\lambda . z & =\lambda \cdot\left(z_{1}+j z_{2}\right)=\lambda z_{1}+j \lambda z_{2}, \\
|.| & : \mathrm{BC} \rightarrow \square, z \rightarrow|z|=\sqrt{\left|z_{1}\right|^{2}+\left|z_{2}\right|^{2}}
\end{aligned}
$$

for all $z=z_{1}+j z_{2}, w=w_{1}+j w_{2} \in \mathrm{BC}$ and for all $\lambda \in \square$.

The subset $\{x+k y: k=i j, x, y \in \square\}$ of $\mathrm{BC}$ is the set of hyperbolic numbers, denoted by $\mathrm{D}$.

Let $z=z_{1}+j z_{2}$ be any bicomplex number in $\mathrm{BC}$. There are three types of conjugates in $\mathrm{BC}$ as follows:

$$
z^{\dagger_{1}}=\bar{z}_{1}+j \overline{z_{2}}, \quad z^{\dagger_{2}}=z_{1}-j z_{2}, \quad z^{\dagger_{3}}=\overline{z_{1}}-j \overline{z_{2}},
$$

where $\overline{z_{1}}, \overline{z_{2}}$ are the complex conjugates of $z_{1}, z_{2} \in \square$. Also, we know three types moduli as follows:

$$
\begin{aligned}
& |z|_{i}^{2}=z z^{\dagger_{2}}=z_{1}^{2}+z_{2}^{2} \in \square, \\
& |z|_{j}^{2}=z z^{\dagger_{1}}=\left(\left|z_{1}\right|^{2}-\left|z_{2}\right|^{2}\right)+j\left(2 \mathfrak{R}\left(z_{1} \cdot \overline{z_{2}}\right)\right), \\
& |z|_{k}^{2}=z z^{\dagger_{3}}=\left(\left|z_{1}\right|^{2}+\left|z_{2}\right|^{2}\right)+k\left(-\mathfrak{J}\left(z_{1} \cdot \overline{z_{2}}\right)\right) \in \mathrm{D} .
\end{aligned}
$$

The numbers $e_{1}=\frac{1+i j}{2}$ and $e_{2}=\frac{1-i j}{2}$ form idempotent basis of bicomplex numbers and hence idempotent representation of any bicomplex number $z=z_{1}+j z_{2}$ is uniquely written as $z=e_{1} \beta_{1}+e_{2} \beta_{2}$ where $\beta_{1}=z_{1}-i z_{2}, \quad \beta_{2}=z_{1}+i z_{2} \in \square$ (Price, 1991).

Let $\alpha=x+k y$ be any hyperbolic number. Then, we have the equality $\quad \alpha=e_{1} \alpha_{1}+e_{2} \alpha_{2}, \quad$ where $\alpha_{1}=x+y, \alpha_{2}=x-y \in \square$. If $\alpha_{1} \geq 0$ and $\alpha_{2} \geq 0$, then $\alpha$ is called a positive hyperbolic number. Therefore, the subset $\left\{\alpha=e_{1} \alpha_{1}+e_{2} \alpha_{2}: \alpha_{1} \geq 0, \alpha_{2} \geq 0\right\}$ of $\mathrm{D}$ is the set of positive hyperbolic numbers, and denoted by $\mathrm{D}^{+}$.

For two hyperbolic numbers $\alpha$ and $\beta$; if their difference $\beta-\alpha \in \mathrm{D}^{+}$(or $\left.\beta-\alpha \in \mathrm{D}^{+}-\{0\}\right)$, then we write $\alpha \precsim \beta$ (or $\alpha \underset{\nabla}{\prec} \beta$ ). For $\alpha=e_{1} \alpha_{1}+e_{2} \alpha_{2}, \beta=\beta_{1} e_{1}+\beta_{2} e_{2} \in \mathrm{D}$ with real numbers $\alpha_{1}, \alpha_{2}, \beta_{1}$ and $\beta_{2}$, we have that

$\alpha \precsim \beta$ if and only if $\alpha_{1} \leq \beta_{1}$ and $\alpha_{2} \leq \beta_{2}$,

$\alpha \prec \beta$ if and only if $\alpha \neq \beta, \alpha_{1} \leq \beta_{1}$ and $\alpha_{2} \leq \beta_{2}$,

$\alpha \prec \beta$ if and only if $\alpha_{1}<\beta_{1}$ and $\alpha_{2}<\beta_{2}$.

The following statements are true for any $\alpha, \beta, \gamma, \delta \in \mathrm{D}, z, w \in \mathrm{BC}$ :

(i) $\quad|z+w|_{k} \precsim|z|_{k}+|w|_{k}, \quad|z w|_{k}=|z|_{k}|w|_{k} \quad$ and $\left|\frac{z}{w}\right|_{k}=\frac{|z|_{k}}{|w|_{k}}$ where $w$ is invertible.

(ii) If $\alpha \in \mathrm{D}^{+}$, then $|\alpha|_{k}=\alpha$ and $|\alpha z|_{k}=\alpha|z|_{k}$.

(iii) $|z|_{k}=\left|\beta_{1}\right| e_{1}+\left|\beta_{2}\right| e_{2}$ for $z=\beta_{1} e_{1}+\beta_{2} e_{2}$.

(iv) If $\alpha \precsim \beta$ and $\gamma \precsim \delta$, then $\alpha+\gamma \precsim \beta+\delta$.

(v) If $\alpha \precsim \beta$ and $0 \precsim \gamma$, then $\alpha \gamma \precsim \beta \gamma$.

(vi) If $\quad \alpha \precsim \beta$ and $\beta \precsim \gamma$, then $\alpha \precsim \gamma \quad$ (LunaElizarrarás et al., 2015). 
(vii) If $\alpha \in \mathrm{D}^{+}, \alpha \neq 1$ and $1-\alpha$ is invertible, then

$$
1+\alpha+\alpha^{2}+\ldots+\alpha^{n}=\frac{1-\alpha^{n+1}}{1-\alpha}
$$

for all $n \in \square$.

(viii) If $\alpha \in \mathrm{D}^{+}$and $\alpha \prec 1$, then $0 \precsim \alpha^{n} \prec 1$ for all $n \in \square$ and $\alpha^{n} \rightarrow 0$ (Sager and Sağır, 2021).

The following concept are recently defined by Kumar and Saini (2016).

Definition 2.1. Let $X$ be a nonempty set and let $d_{\mathrm{D}}: X \times X \rightarrow \mathrm{D}$ be a function such that the following properties hold:

(i) $0 \precsim d_{\mathrm{D}}(x, y)$, and $d_{\mathrm{D}}(x, y)=0$ if and only if $x=y$.

(ii) $d_{\mathrm{D}}(x, y)=d_{\mathrm{D}}(y, x)$.

(iii) $d_{\mathrm{D}}(x, z) \precsim d_{\mathrm{D}}(x, y)+d_{\mathrm{D}}(y, z)$

for any $x, y, z \in X$. Then, $d_{\mathrm{D}}$ is said to be a $\mathrm{D}-$ valued or hyperbolic valued metric on $X$ and the pair $\left(X, d_{\mathrm{D}}\right)$ is said to be a hyperbolic valued or $\mathrm{D}$-valued metric space.

Definition 2.2. (Zeyada et al., 2006) Let $X$ be a nonempty set and let $d: X \times X \rightarrow[0, \infty)$ be a function satisfying the following conditions:

(i) If $d(x, y)=0$, then $x=y$.

(ii) $d(x, y)=d(y, x)$.

(iii) $d(x, z) \leq d(x, y)+d(y, z)$

\section{Results}

Now, we give the following definitions at the beginning of this section.

Definition 3.1. Let $X$ be a nonempty set and let $d_{\mathrm{D}}^{d i s}: X \times X \rightarrow \mathrm{D}^{+}$be a mapping such that the following properties hold:

(i) If $d_{\mathrm{D}}^{\text {dis }}(x, y)=0$, then $x=y$.

(ii) $d_{\mathrm{D}}^{\text {dis }}(x, y)=d_{\mathrm{D}}^{\text {dis }}(y, x)$.

(iii) $d_{\mathrm{D}}^{\text {dis }}(x, z) \precsim d_{\mathrm{D}}^{\text {dis }}(x, y)+d_{\mathrm{D}}^{d i s}(y, z)$

for any $x, y, z \in X$. Then, $d_{\mathrm{D}}^{\text {dis }}$ is said to be a hyperbolic valued dislocated metric or $\mathrm{D}$-valued $d$-metric on $X$ and for all $x, y, z \in X$. Then, $d$ is said to be a dislocated metric or $d$-metric on $X$ and the pair $(X, d)$ is said to be a dislocated metric or $d$-metric space.

It is evident that metric spaces are $d$-metric spaces. However the converse may not always be true by the next example.

Example 2.3. (Rahman and Sarwar, 2015) Let $d:[0, \infty) \times[0, \infty) \rightarrow[0, \infty)$ be defined by $d(x, y)=\max \{x, y\}$. It is simple to show that $d$ is a $d-$ metric but not a metric.

Definition 2.4. (Hitzler and Seda, 2000) Let $(X, d)$ be a $d-$ metric space. A mapping $T: X \rightarrow X$ is said to be a contraction if there exists $0 \leq \alpha<1$ such that

$$
d(T x, T y) \leq \alpha d(x, y)
$$

for all $x, y \in X$.

Theorem 2.5. (Hitzler and Seda, 2000) Let $(X, d)$ be a complete $d$-metric space and let $T: X \rightarrow X$ be a contraction. Then, $T$ has a unique fixed point.

the pair $\left(X, d_{\mathrm{D}}^{\text {dis }}\right)$ is said to be a hyperbolic valued dislocated metric or $\mathrm{D}$ - valued $d$-metric space.

Definition 3.2. Let $\left(X, d_{\mathrm{D}}^{\text {dis }}\right)$ be a $\mathrm{D}$-valued $d$-metric space, $\left(x_{n}\right)$ be any sequence in $X$ and $x \in X$. If for every $0 \prec \varepsilon \in \mathrm{D}$ there exists $n_{0} \in \square$ depending on $\varepsilon$ such that $d_{\mathrm{D}}^{\text {dis }}\left(x_{n}, x\right) \prec \varepsilon$ for all $n \geq n_{0}$ then we say that $\left(x_{n}\right)$ is $d_{\mathrm{D}}^{d i s}$ - convergent, and denoted by $x_{n} \stackrel{d_{\mathrm{D}}^{d i s}}{\rightarrow} x$ as $n \rightarrow \infty$.

If for every $0 \prec \varepsilon \in \mathrm{D}$ there exists $n_{0} \in \square$ depending on $\varepsilon$ such that $d_{\mathrm{D}}^{d i s}\left(x_{n}, x_{m}\right) \prec \varepsilon$ for all $n, m \geq n_{0}$ then we say that $\left(x_{n}\right)$ is a $d_{\mathrm{D}}^{d i s}-$ Cauchy sequence. 
If every $d_{\mathrm{D}}^{d i s}$ - Cauchy sequence is $d_{\mathrm{D}}^{d i s}$-convergent in $\left(X, d_{\mathrm{D}}^{d i s}\right)$, then we say that $\left(X, d_{\mathrm{D}}^{d i s}\right)$ is a complete $\mathrm{D}-$ valued $d$-metric space.

Definition 3.3. Let $\left(X, d_{\mathrm{D}}^{d i s}\right)$ be a hyperbolic valued dislocated metric space. A mapping $T: X \rightarrow X$ is is said to be a $\mathrm{D}-$ valued $d$-contraction if there exists $0 \precsim \alpha \prec 1$ such that

$$
d_{\mathrm{D}}^{d i s}(T x, T y) \precsim \alpha d_{\mathrm{D}}^{d i s}(x, y)
$$

for all $x, y \in X$ where the hyperbolic number $1-\alpha$ is invertible.

Based on the idea of the Banach's fixed point theorem (Banach, 1922) in classical metric spaces, we establish the following main theorem which implies the existence and uniqueness of a fixed point on complete hyperbolic valued dislocated metric spaces.

Theorem 3.4. Let $\left(X, d_{\mathrm{D}}^{\text {dis }}\right)$ be a complete hyperbolic valued dislocated metric space and $T$ be a $\mathrm{D}$ - valued $d$-contraction on $X$. Then, $T$ has a unique fixed point.

Proof. Choose $x_{0} \in X$ arbitrarily. There exists $x_{1} \in X$ such that $T x_{0}=x_{1}$. Continuing in the same manner, we construct the sequence $\left(x_{n}\right)$ in $X$ such that $T x_{n}=x_{n+1}$.

Let $m, n \in \square$ and $m<n$. Then, by a similar way to proof of main theorem in (Sager and Sağır, 2021), we obtain the inequality

$$
d_{\mathrm{D}}^{d i s}\left(x_{m}, x_{n}\right) \prec \alpha^{m} d_{\mathrm{D}}^{d i s}\left(x_{0}, x_{1}\right) \frac{1}{1-\alpha}
$$

and so $\left(x_{n}\right)$ is a $d_{\mathrm{D}}^{\text {dis }}-$ Cauchy sequence. The completenes of $X$ explains that there is a point $x \in X$ such that $x_{n} \stackrel{d_{\mathrm{D}}^{d i s}}{\rightarrow} x$ as $n \rightarrow \infty$.

Now, we prove that $x=T x$. Suppose this is not true: $x \neq T x$. Taking $d_{\mathrm{D}}^{\text {dis }}(x, T x)=\varepsilon$ we set

$$
B=\left\{z \in X: d_{\mathrm{D}}^{d i s}(x, z) \precsim\left(\frac{1}{4} e_{1}+\frac{1}{4} e_{2}\right) \varepsilon\right\} .
$$

For $z \in B$, we write

$$
\begin{aligned}
\varepsilon & =d_{\mathrm{D}}^{d i s}(x, T x) \precsim d_{\mathrm{D}}^{d i s}(x, z)+d_{\mathrm{D}}^{d i s}(z, T x) \\
& \precsim\left(\frac{1}{4} e_{1}+\frac{1}{4} e_{2}\right) \varepsilon+d_{\mathrm{D}}^{d i s}(z, T x)
\end{aligned}
$$

and so $\left(\frac{3}{4} e_{1}+\frac{3}{4} e_{2}\right) \varepsilon \precsim d_{\mathrm{D}}^{d i s}(z, T x)$.

On the other hand, since $\left(x_{n}\right) d_{\mathrm{D}}^{\text {dis }}-$ converges to $x$, there is a natural number $n_{0}$ such that $d_{\mathrm{D}}^{\text {dis }}\left(x_{n}, x\right) \precsim\left(\frac{1}{4} e_{1}+\frac{1}{4} e_{2}\right) \varepsilon$ for all $n \geq n_{0}$. This means $x_{n} \in B$ for all $n \geq n_{0}$. Then, by (3.1) we have

$$
\begin{aligned}
d_{\mathrm{D}}^{d i s}\left(x_{n_{0}+1}, T x\right) & =d_{\mathrm{D}}^{d i s}\left(T x_{n_{0}}, T x\right) \precsim \alpha d_{\mathrm{D}}^{d i s}\left(x_{n_{0}}, x\right) \\
& \prec d_{\mathrm{D}}^{d i s}\left(x_{n_{0}}, x\right) \precsim\left(\frac{1}{4} e_{1}+\frac{1}{4} e_{2}\right) \varepsilon \\
& \prec\left(\frac{3}{4} e_{1}+\frac{3}{4} e_{2}\right) \varepsilon \precsim d_{\mathrm{D}}^{\text {dis }}\left(x_{n_{0}+1}, T x\right)
\end{aligned}
$$

and this is a contradiction. Hence $x=T x$. This implies that $x$ is a fixed point of the mapping $T$.

To show that the fixed point is unique, suppose that there is an element $x^{*} \neq x$ in $X$ such that $T x^{*}=x^{*}$. Then, we have $d_{\mathrm{D}}^{\text {dis }}\left(T x, T x^{*}\right)=d_{\mathrm{D}}^{d i s}\left(x, x^{*}\right)$. This shows that the condition (3.1) is not satisfied. But this is a contradiction, hence fixed point is unique.

Example 3.5. Define a mapping $d_{\mathrm{D}}^{d i s}: \mathrm{D}^{+} \times \mathrm{D}^{+} \rightarrow \mathrm{D}^{+}$as

$$
d_{\mathrm{D}}^{\text {dis }}(\alpha, \beta)=\max \left\{\alpha_{1}, \beta_{1}\right\} e_{1}+\max \left\{\alpha_{2}, \beta_{2}\right\} e_{2},
$$

where $\alpha=\alpha_{1} e_{1}+\alpha_{2} e_{2}, \beta=\beta_{1} e_{1}+\beta_{2} e_{2} \in \mathrm{D}^{+}$. Then, it can be easily showed that $\left(\mathrm{D}^{+}, d_{\mathrm{D}}^{\text {dis }}\right)$ is a complete $\mathrm{D}$ - valued $d$ - metric space.

Consider a mapping $T$ on $\mathrm{D}^{+}$with $\gamma=\gamma_{1} e_{1}+\gamma_{2} e_{2}$ as

$$
T \gamma= \begin{cases}\frac{2 \gamma_{1}+\left(\gamma_{1}\right)^{3}}{9} e_{1}+\frac{2 \gamma_{2}+\left(\gamma_{2}\right)^{3}}{9} e_{2}, \quad \gamma_{1}, \gamma_{2} \in[0,1) \\ \frac{2 \gamma_{1}+\left(\gamma_{1}\right)^{3}}{9} e_{1}+\frac{\gamma_{2}}{17} e_{2}, & \gamma_{1} \in[0,1), \gamma_{2} \in[1, \infty) \\ \frac{\gamma_{1}}{17} e_{1}+\frac{2 \gamma_{2}+\left(\gamma_{2}\right)^{3}}{9} e_{2}, & \gamma_{1} \in[1, \infty), \gamma_{2} \in[0,1) \\ \frac{\gamma_{1}}{17} e_{1}+\frac{\gamma_{2}}{17} e_{2}, & \gamma_{1} \in[1, \infty), \gamma_{2} \in[1, \infty)\end{cases}
$$

Let $\alpha_{1}, \alpha_{2} \in[0,1)$ and $\beta_{1}, \beta_{2} \in[1, \infty)$. Then, we have 


$$
\begin{aligned}
& d_{\mathrm{D}}^{d i s}(T \alpha, T \beta) \\
& =d_{\mathrm{D}}^{d i s}\left(\frac{2 \alpha_{1}+\left(\alpha_{1}\right)^{3}}{9} e_{1}+\frac{2 \alpha_{2}+\left(\alpha_{2}\right)^{3}}{9} e_{2}, \frac{\beta_{1}}{17} e_{1}+\frac{\beta_{2}}{17} e_{2}\right) \\
& =\max \left\{\frac{2 \alpha_{1}+\left(\alpha_{1}\right)^{3}}{9}, \frac{\beta_{1}}{17}\right\} e_{1}+\max \left\{\frac{2 \alpha_{2}+\left(\alpha_{2}\right)^{3}}{9}, \frac{\beta_{2}}{17}\right\} e_{2} \\
& \precsim \max \left\{\frac{\alpha_{1}}{3}, \frac{\beta_{1}}{17}\right\} e_{1}+\max \left\{\frac{\alpha_{2}}{3}, \frac{\beta_{2}}{17}\right\} e_{2} \\
& \precsim \frac{1}{3} \max \left\{\alpha_{1}, \beta_{1}\right\} e_{1}+\frac{1}{3} \max \left\{\alpha_{2}, \beta_{2}\right\} e_{2} \\
& =\left(\frac{1}{3} e_{1}+\frac{1}{3} e_{2}\right) d_{\mathrm{D}}^{d i s}(\alpha, \beta) .
\end{aligned}
$$

Also, the other cases for $\alpha, \beta \in \mathrm{D}^{+}$can be easily proved in a similar way as above.

Then, the inequality

$$
d_{\mathrm{D}}^{d i s}(T \alpha, T \beta) \precsim\left(\frac{1}{3} e_{1}+\frac{1}{3} e_{2}\right) d_{\mathrm{D}}^{d i s}(\alpha, \beta)
$$

holds for all $\alpha, \beta \in \mathrm{D}^{+}$. Thus, the mapping $T$ is a $\mathrm{D}-$ valued $d$-contraction mapping since $0 \prec \frac{1}{3} e_{1}+\frac{1}{3} e_{2} \prec 1 \quad$ and $1-\left(\frac{1}{3} e_{1}+\frac{1}{3} e_{2}\right)=\frac{2}{3} e_{1}+\frac{2}{3} e_{2}$ is invertible. Therefore, $T$ has a fixed point $\gamma=0 \in \mathrm{D}^{+}$, which is unique.

The following result is a new version of Kannan's fixed point theorem (Kannan, 1968) in hyperbolic valued dislocated metric spaces.

Theorem 3.6. Let $\left(X, d_{\mathrm{D}}^{d i s}\right)$ be a complete hyperbolic valued dislocated metric space and $T: X \rightarrow X$ be a mapping. If there exists $0 \precsim \alpha \prec \frac{1}{2}$ such that

$$
d_{\mathrm{D}}^{d i s}(T x, T y) \precsim \alpha\left[d_{\mathrm{D}}^{d i s}(x, T x)+d_{\mathrm{D}}^{d i s}(y, T y)\right]
$$

for all $x, y \in X$ where the hyperbolic number $1-\alpha$ is invertible, then $T$ has a unique fixed point.

Proof. Choose $x_{0} \in X$ arbitrarily. Consider the sequence $\left(x_{n}\right)$ in $X$ such that $T x_{0}=x_{1}$ and $T x_{n}=x_{n+1}$.

By (3.2), we have

$$
\begin{aligned}
d_{\mathrm{D}}^{\text {dis }}\left(x_{n}, x_{n+1}\right) & =d_{\mathrm{D}}^{\text {dis }}\left(T x_{n-1}, T x_{n}\right) \\
& \precsim \alpha\left[d_{\mathrm{D}}^{\text {dis }}\left(x_{n-1}, T x_{n-1}\right)+d_{\mathrm{D}}^{\text {dis }}\left(x_{n}, T x_{n}\right)\right] \\
& =\alpha\left[d_{\mathrm{D}}^{\text {dis }}\left(x_{n-1}, x_{n}\right)+d_{\mathrm{D}}^{\text {dis }}\left(x_{n}, x_{n+1}\right)\right]
\end{aligned}
$$

and so

$$
\begin{aligned}
d_{\mathrm{D}}^{d i s}\left(x_{n}, x_{n+1}\right) & \precsim \frac{\alpha}{1-\alpha} d_{\mathrm{D}}^{\operatorname{dis}}\left(x_{n-1}, x_{n}\right) \\
& \precsim\left(\frac{\alpha}{1-\alpha}\right)^{2} d_{\mathrm{D}}^{d i s}\left(x_{n-2}, x_{n-1}\right) \\
& \vdots \\
& \precsim\left(\frac{\alpha}{1-\alpha}\right)^{n} d_{\mathrm{D}}^{\operatorname{dis}}\left(x_{0}, x_{1}\right) .
\end{aligned}
$$

For $m, n \in \square$ and $m>n$, we write

$$
\begin{aligned}
d_{\mathrm{D}}^{d i s}\left(x_{n}, x_{m}\right) \precsim & d_{\mathrm{D}}^{d i s}\left(x_{n}, x_{n+1}\right)+d_{\mathrm{D}}^{d i s}\left(x_{n+1}, x_{n+2}\right)+\ldots+d_{\mathrm{D}}^{d i s}\left(x_{m-1}, x_{m}\right) \\
& \precsim\left[\left(\frac{\alpha}{1-\alpha}\right)^{n}+\left(\frac{\alpha}{1-\alpha}\right)^{n+1}+\ldots+\left(\frac{\alpha}{1-\alpha}\right)^{m-1}\right] d_{\mathrm{D}}^{d i s}\left(x_{0}, x_{1}\right) \\
& =\left(\frac{\alpha}{1-\alpha}\right)^{n}\left[1+\left(\frac{\alpha}{1-\alpha}\right)+\ldots+\left(\frac{\alpha}{1-\alpha}\right)^{m-n-1}\right] d_{\mathrm{D}}^{d i s}\left(x_{0}, x_{1}\right) \\
& =\left(\frac{\alpha}{1-\alpha}\right)^{n} \frac{1-\left(\frac{\alpha}{1-\alpha}\right)^{m-n}}{1-\frac{\alpha}{1-\alpha}} d_{\mathrm{D}}^{d i s}\left(x_{0}, x_{1}\right) \\
& \prec \frac{\left(\frac{\alpha}{1-\alpha}\right)^{n}}{1-\frac{\alpha}{1-\alpha}} d_{\mathrm{D}}^{d i s}\left(x_{0}, x_{1}\right) .
\end{aligned}
$$

Since $d_{\mathrm{D}}^{\text {dis }}\left(x_{0}, x_{1}\right) \in \mathrm{D}^{+}$is fixed and $\left(\frac{\alpha}{1-\alpha}\right)^{n} \prec 1$, we can make $\frac{\left(\frac{\alpha}{1-\alpha}\right)^{n}}{1-\frac{\alpha}{1-\alpha}} d_{\mathrm{D}}^{\text {dis }}\left(x_{0}, x_{1}\right)$ as small as we want by taking $n$ sufficiently large. It follows that $\left(x_{n}\right)$ is a $d_{\mathrm{D}}^{\text {dis }}-$ Cauchy sequence. By the completeness of $X$, there is a point $x \in X$ such that $x_{n} \stackrel{d_{\mathrm{D}}^{d i s}}{\rightarrow} x$ as $n \rightarrow \infty$.

Now, we prove that $x=T x$. Then, we have

$$
\begin{aligned}
& d_{\mathrm{D}}^{d i s}(x, T x) \\
& \precsim d_{\mathrm{D}}^{d i s}\left(x, x_{n}\right)+d_{\mathrm{D}}^{d i s}\left(x_{n}, T x\right) \\
& =d_{\mathrm{D}}^{d i s}\left(x, x_{n}\right)+d_{\mathrm{D}}^{d i s}\left(T x_{n-1}, T x\right) \\
& \precsim d_{\mathrm{D}}^{d i s}\left(x, x_{n}\right)+\alpha\left(\frac{\alpha}{1-\alpha}\right)^{n-1} d_{\mathrm{D}}^{d i s}\left(x_{0}, x_{1}\right)+\alpha d_{\mathrm{D}}^{d i s}(x, T x),
\end{aligned}
$$


and so

$$
d_{\mathrm{D}}^{d i s}(x, T x) \precsim \frac{1}{1-\alpha} d_{\mathrm{D}}^{d i s}\left(x, x_{n}\right)+\left(\frac{\alpha}{1-\alpha}\right)^{n} d_{\mathrm{D}}^{d i s}\left(x_{0}, x_{1}\right) .
$$

Therefore we obtain that $d_{\mathrm{D}}^{\text {dis }}(x, T x)=0$ letting $n \rightarrow \infty$ hence $T x=x$. This shows that $x$ is a fixed point of the mapping $T$.

Also, for any fixed point $x$ of the mapping $T$ we get

$$
\begin{aligned}
d_{\mathrm{D}}^{d i s}(x, x) & =d_{\mathrm{D}}^{d i s}(T x, T x) \\
& \precsim \alpha\left[d_{\mathrm{D}}^{d i s}(x, T x)+d_{\mathrm{D}}^{d i s}(x, T x)\right] \\
& =\alpha\left[d_{\mathrm{D}}^{d i s}(x, x)+d_{\mathrm{D}}^{d i s}(x, x)\right] \\
& =2 \alpha d_{\mathrm{D}}^{d i s}(x, x)
\end{aligned}
$$

and $\quad$ so, $\quad(1-2 \alpha) d_{\mathrm{D}}^{d i s}(x, x) \precsim 0$. This implies that $d_{\mathrm{D}}^{d i s}(x, x)=0$ since $0 \precsim \alpha \prec \frac{1}{2}$.

To show that the fixed point is unique, we assume that there is an element $x^{*} \neq x$ in $X$ such that $T x^{*}=x^{*}$. Then, we have

$$
\begin{aligned}
d_{\mathrm{D}}^{d i s}\left(x, x^{*}\right) & =d_{\mathrm{D}}^{d i s}\left(T x, T x^{*}\right) \\
& \precsim \alpha\left[d_{\mathrm{D}}^{d i s}(x, T x)+d_{\mathrm{D}}^{d i s}\left(x^{*}, T x^{*}\right)\right] . \\
& =\alpha\left[d_{\mathrm{D}}^{d i s}(x, x)+d_{\mathrm{D}}^{d i s}\left(x^{*}, x^{*}\right)\right] \\
& =0
\end{aligned}
$$

But this is impossible, hence fixed point is unique.

We now state another theorem which is an extension of Chatterjea's fixed point result (Chatterjea, 1972) for selfmappings on hyperbolic valued dislocated metric spaces as follows:

Theorem 3.7. Let $\left(X, d_{\mathrm{D}}^{\text {dis }}\right)$ be a complete hyperbolic valued dislocated metric space and $T: X \rightarrow X$ be a continuous mapping. If there exists $0 \precsim \alpha \prec \frac{1}{4}$ such that

$$
d_{\mathrm{D}}^{d i s}(T x, T y) \precsim \alpha\left[d_{\mathrm{D}}^{d i s}(x, T y)+d_{\mathrm{D}}^{d i s}(y, T x)\right]
$$

for all $x, y \in X$ where the hyperbolic number $1-\alpha$ is invertible, then $T$ has a unique fixed point.

Proof. Choose $x_{0} \in X$ arbitrarily. Consider the sequence $\left(x_{n}\right)$ in $X$ such that $T x_{0}=x_{1}$ and $T x_{n}=x_{n+1}$.

By (3.3), we have

$$
\begin{aligned}
& d_{\mathrm{D}}^{d i s}\left(x_{n}, x_{n+1}\right) \\
& =d_{\mathrm{D}}^{d i s}\left(T x_{n-1}, T x_{n}\right) \\
& \precsim \alpha\left[d_{\mathrm{D}}^{d i s}\left(x_{n-1}, T x_{n}\right)+d_{\mathrm{D}}^{d i s}\left(x_{n}, T x_{n-1}\right)\right] \\
& =\alpha\left[d_{\mathrm{D}}^{d i s}\left(x_{n-1}, x_{n+1}\right)+d_{\mathrm{D}}^{d i s}\left(x_{n}, x_{n}\right)\right] \\
& \precsim \alpha\left[d_{\mathrm{D}}^{d i s}\left(x_{n-1}, x_{n}\right)+d_{\mathrm{D}}^{d i s}\left(x_{n}, x_{n+1}\right)+d_{\mathrm{D}}^{d i s}\left(x_{n}, x_{n-1}\right)+d_{\mathrm{D}}^{d i s}\left(x_{n-1}, x_{n}\right)\right] \\
& =\alpha d_{\mathrm{D}}^{d i s}\left(x_{n}, x_{n+1}\right)+3 \alpha d_{\mathrm{D}}^{d i s}\left(x_{n-1}, x_{n}\right)
\end{aligned}
$$

and so

$$
\begin{aligned}
& d_{\mathrm{D}}^{d i s}\left(x_{n}, x_{n+1}\right) \precsim \frac{3 \alpha}{1-\alpha} d_{\mathrm{D}}^{d i s}\left(x_{n-1}, x_{n}\right) \\
& \precsim\left(\frac{3 \alpha}{1-\alpha}\right)^{2} d_{\mathrm{D}}^{d i s}\left(x_{n-2}, x_{n-1}\right) \\
& \precsim\left(\frac{3 \alpha}{1-\alpha}\right)^{n} d_{\mathrm{D}}^{d i s}\left(x_{0}, x_{1}\right)
\end{aligned}
$$

Let $m, n \in \square$ and $m>n$. Thus, we can write

$$
\begin{aligned}
d_{\mathrm{D}}^{d i s}\left(x_{n}, x_{m}\right) \precsim & d_{\mathrm{D}}^{d i s}\left(x_{n}, x_{n+1}\right)+d_{\mathrm{D}}^{d i s}\left(x_{n+1}, x_{n+2}\right)+\ldots+d_{\mathrm{D}}^{d i s}\left(x_{m-1}, x_{m}\right) \\
& \precsim\left[\left(\frac{3 \alpha}{1-\alpha}\right)^{n}+\left(\frac{3 \alpha}{1-\alpha}\right)^{n+1}+\ldots+\left(\frac{3 \alpha}{1-\alpha}\right)^{m-1}\right] d_{\mathrm{D}}^{d i s}\left(x_{0}, x_{1}\right) \\
& =\left(\frac{3 \alpha}{1-\alpha}\right)^{n}\left[1+\left(\frac{3 \alpha}{1-\alpha}\right)^{n}+\ldots+\left(\frac{3 \alpha}{1-\alpha}\right)^{m-n-1}\right] d_{\mathrm{D}}^{d i s}\left(x_{0}, x_{1}\right) \\
& =\left(\frac{3 \alpha}{1-\alpha}\right)^{n} \frac{1-\left(\frac{3 \alpha}{1-\alpha}\right)^{m-n}}{1-\frac{3 \alpha}{1-\alpha}} d_{\mathrm{D}}^{d i s}\left(x_{0}, x_{1}\right) \\
& \prec \frac{\left(\frac{3 \alpha}{1-\alpha}\right)^{n}}{1-\frac{3 \alpha}{1-\alpha}} d_{\mathrm{D}}^{d i s}\left(x_{0}, x_{1}\right) .
\end{aligned}
$$

Similarly as in the proof of Theorem 3.6, it follows that $\left(x_{n}\right)$ is a $d_{\mathrm{D}}^{d i s}$ - Cauchy sequence, and so there exists a point $x \in X$ $d_{\mathrm{D}}^{\text {dis }}$

such that $x_{n} \rightarrow x$ as $n \rightarrow \infty$ by the completeness of $X$.

On the other hand, since $X$ is a continuous mapping, $d_{\mathrm{D}}^{\text {dis }}$ $x_{n} \rightarrow x$ as $n \rightarrow \infty$ and $x_{n+1}=T x$, we deduce that $T x=x$, that is, $x$ is a fixed point of the mapping $T$.

To prove that this fixed point is unique, we assume that there is an element $x^{*} \neq x$ in $X$ such that $T x^{*}=x^{*}$. Then, we have 


$$
\begin{aligned}
d_{\mathrm{D}}^{d i s}\left(x, x^{*}\right) & =d_{\mathrm{D}}^{d i s}\left(T x, T x^{*}\right) \\
& \precsim \alpha\left[d_{\mathrm{D}}^{d i s}\left(x, T x^{*}\right)+d_{\mathrm{D}}^{d i s}\left(x^{*}, T x\right)\right] \\
& =\alpha\left[d_{\mathrm{D}}^{d i s}\left(x, x^{*}\right)+d_{\mathrm{D}}^{d i s}\left(x^{*}, x\right)\right] \\
& =2 \alpha d_{\mathrm{D}}^{d i s}\left(x, x^{*}\right)
\end{aligned}
$$

and so, $\quad(1-2 \alpha) d_{\mathrm{D}}^{\text {dis }}\left(x, x^{*}\right) \precsim 0$. This implies that $d_{\mathrm{D}}^{d i s}\left(x, x^{*}\right)=0$ since $0 \precsim \alpha \prec \frac{1}{4}$. But this is impossible, hence fixed point is unique.

\section{Conclusion}

In this study, inspired by the ideas of dislocated metric spaces and hyperbolic valued metric spaces, we introduce hyperbolic valued dislocated metric spaces, and we state some fixed point theorems for self-mappings with Banach, Kannan and Chatterjea type contractions on such spaces. Our new findings carry some well known results from the recent literature to hyperbolic valued dislocated metric spaces. Using different contractive conditions, it can be established new fixed point results for hyperbolic valued dislocated metric spaces.

\section{References}

Banach, S. (1922). Sur les opérations dans les ensembles abstraits et leur application aux équations intégrales. Fund. Math., 3(1), 133-181.

Chatterjea, S. K. (1972). Fixed-point theorems. C. R. Acad. Bulgare Sci., 25, 727-730.

Gaba H. \& Garg A. K. (2019). Some fixed point results for contraction in dislocated metric space. International Journal on Emerging Technologies, 10(2b), 147-150.

Hitzler, P., \& Seda, A. K. (2000). Dislocated topologies. J. Electr. Eng., 51(12), 3-7.

Kannan, R. (1968). Some results on fixed points. Bull. Cal. Math. Soc., 60, 71-76.

Kumar, R., \& Saini, H. (2016). Topological bicomplex modules. Advances in Applied Clifford Algebras, 26(4), 1249-1270.

Luna-Elizarrarás, M. E., Shapiro, M., Struppa, D. C., \& Vajiac, A. (2015). Bicomplex holomorphic functions: the algebra, geometry and analysis of bicomplex numbers. Birkhäuser.

Pasicki, L. (2015). Dislocated metric and fixed point theorems. Fixed Point Theory and Applications, 2015(1), 1-14.

Price, G. B. (1991). An introduction to multicomplex spaces and functions. M. Dekker.

Rahman, M. U., \& Sarwar, M. (2015). Fixed point theorems for expanding mappings in dislocated metric space. Mathematical Sciences Letters, 4(1), 69-73.

Sager, N., \& Sağır, B. (2021). Fixed points of hyperbolic contraction mappings on hyperbolic valued metric spaces. Sarajevo Journal of Mathematics, 17(2). (in print)

Sager, N., \& Sağır, B. (2021). Common fixed, coupled coincidence and common coupled fixed point results in hyperbolic valued metric spaces. Boletim da Sociedade Paranaense de Matemática. (accepted for publication)

Wadkar, B. R., Bhardwaj, R., \& Singh, B. (2017). Some fixed point theorems in dislocated metric space. Global Journal of Pure and Applied Mathematics, 13(6), 2089-2110.
Zeyada, F. M., Hassan, G. H., \& Ahmed, M. A. (2006). A generalization of a fixed point theorem due to Hitzler and Seda in dislocated quasi-metric spaces. Arabian Journal for Science and Engineering, 31(1A), 111-114. 